\title{
Latente Tuberkulose-Infektion: 12-wöchige Therapie mit Rifapentin und Isoniazid zur Prävention ab 2 Jahren von der FDA zugelassen
}

Hintergrund Kinder profitieren von der präventiven Therapie einer latenten Tuberkulose (TB)-Infektion, da ihr Erkrankungsrisiko höher ist als das von Erwachsenen und häufiger ein schwerer Verlauf mit Meningitis oder Dissemination auftritt. Eine prospektive offene randomisierte Studie an 7731 Patienten mit latenter TB-Infektion konnte 2011 zeigen, dass eine direkt überwachte 3-monatige Therapie mit wöchentlicher Gabe von $900 \mathrm{mg}$ Rifapentin und $900 \mathrm{mg}$ Isoniazid (INH) mind. ebenso gut wirksam ist wie die 9-monatige tägliche Einnahme von INH $300 \mathrm{mg}$ [1]. Die Rate der TB-Entwicklung war $0,1 \%$ in der Kombinations- und 0,2\% in der INH-Monotherapiegruppe. Einen vollständigen Therapieabschluss erreichten $82 \%$ der Patienten unter der Kombinations- und 69\% unter INH-Monotherapie. Der Therapieabbruch erfolgte aufgrund von Nebenwirkungen bei 4,9\% (Kombination) bzw. bei 3,7\% (INH-Mono). Die Hepatotoxizität war in der Kombinationsgruppe seltener ( 0,4 vs. 2,7\%), eine Hypersensitivitätsreaktion häufiger (2,9 vs. $0,4 \%$ ).

Methode Kinder und Jugendliche mit latenter TB wurden in einer Therapiestudie von Juni 2001 bis Dezember 2010 an 29 Studienzentren in den USA, Kanada,
Brasilien, Hong Kong und Spanien untersucht. Die Verlaufsbeobachtung erfolgte bis September 2013. In diese Studie wurden zunächst Kinder ab 12 Jahren eingeschlossen, die entweder mit 12 direkt überwachten wöchentlichen Gaben von $900 \mathrm{mg}$ Rifapentin und $900 \mathrm{mg}$ INH über 3 Monate oder mit 270 täglichen nicht überwachten Gaben von $300 \mathrm{mg}$ INH über 9 Monate behandelt wurden. Nachdem im Jahr 2005 Daten zur Pharmakokinetik von Rifapentin bei jüngeren Kindern vorlagen, wurden zusätzlich Kinder ab 2 Jahren eingeschlossen. Das Auftreten einer TB wurde bis 33 Monate nach Einschluss beobachtet.

Ergebnisse Insgesamt wurden 1058 Kinder zwischen 2 und 17 Jahren behandelt, von 905 konnten die Daten ausgewertet werden. In der Kombinationsgruppe beendeten $88,1 \%$ die Behandlung, in der INHGruppe nur 80,9\% ( $p=0,003)$. Aufgrund von Nebenwirkungen brachen unter Kombinationstherapie 8 Kinder (1,7\%) die Studie ab, 3 wegen grippeartiger Symptome, 3 wegen Hauterkrankungen mit juckenden Ekzemen, eines davon zusätzlich mit Fieber und Mundschleimhautbläschen, und 2 aufgrund gastrointestinaler Nebenwirkungen. In der INH-Gruppe brachen 2 Kinder $(0,5 \%)$ wegen Hautreaktionen und gastro- intestinaler Nebenwirkungen die Studie ab. In keiner Gruppe traten hepatotoxische oder schwerwiegende Nebenwirkungen sowie therapieassoziierte Todesfälle auf. 5 Kinder hatten eine HIV-Koinfektion, keines davon hatte schwerwiegende Nebenwirkungen. In der Kombinationstherapiegruppe entwickelte kein Kind eine TB, in der Isoziazidgruppe 3 von 434 Kindern (kumulative Rate $0,74 \%)$.

Schlussfolgerung $\mathrm{Ab}$ einem Alter von 2 Jahren ist die 3-monatige Therapie mit wöchentlicher Gabe von 900 mg Rifapentin und INH ebenso sicher und wirksam wie die 9-monatige tägliche Einnahme von INH $300 \mathrm{mg}$. Die Therapieadhärenz ist besser.

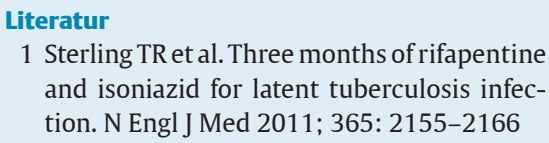

1 Sterling TR et al. Three months of rifapentine and isoniazid for latent tuberculosis infection. N Engl J Med 2011; 365: 2155-2166

Villarino ME, Scott NA, Weis SE et al. Treatment for preventing tuberculosis in children and adolescents: A randomized clinical trial of a 3-month, 12-dose regimen of a combination of Rifapentine and Isoniazid.

JAMA Pediatr 2015; 169: 247-255

Kommentar

Mit dieser Studie wird die Wirksamkeit und Sicherheit der Therapie bei Kindern ab 2 Jahren nachgewiesen. Eine gewisse Verzerrung der Ergebnisse zugunsten der Kombinationstherapie ist durch das Studiendesign möglich, da nur die Kombinationstherapie direkt überwacht wurde, während die INH-Therapie ohne Überwachung erfolgte. Rifapentin ist ein Rifamycinderivat mit einer stärkeren bakteriziden Wirkung gegen Mycobacterium tuberculosis und einer längeren Halbwertszeit als Rifampicin. Bereits 2010 wurde es als Orphan-Drug zur TB-Therapie zugelassen. Eine bekannte Nebenwirkung ist die Rotfärbung von Körperflüssigkeiten einschließlich Urin und Tränenflüssigkeit, die zur Verfärbung von Kontaktlinsen führen kann. Neutropenie und Transaminasenerhöhung sind seltene Nebenwirkungen.
Hypersensitivitätsreaktionen mit Symptomen wie Kopfschmerzen, Schwindel, muskuloskelettale Schmerzen, Petechien, Purpura und Juckreiz können auftreten. Rifapentin wird über Cytochrom P450 3A metabolisiert, so dass es zu Interaktionen z. B. mit Phenprocoumon, Methadon und oralen Kontrazeptiva kommt. Dosierung der Kombinationstherapie: Isoniazid $15 \mathrm{mg} / \mathrm{kg}$ Körpergewicht, aufgerundet auf die nächstgelegenen 50 oder $100 \mathrm{mg}$ (max. $900 \mathrm{mg}$ ); Rifapentin $300 \mathrm{mg}$ bis $14 \mathrm{~kg}$ Körpergewicht, $450 \mathrm{mg}$ bis $25 \mathrm{~kg}, 600 \mathrm{mg}$ bis $32 \mathrm{~kg}, 750 \mathrm{mg}$ bis $50 \mathrm{~kg}$ sowie über $50 \mathrm{~kg}$ Körpergewicht die max. Dosis von $900 \mathrm{mg}$. Im Dezember 2014 wurde Rifapentin für die Behandlung der latenten TB-Infektion in Kombination mit INH von der US-amerikanischen Food and Drug Administration (FDA) ab einem Alter von 2 Jahren zugelas- sen. Rifapentin ist derzeit nur in den USA erhältlich und kann über die Internationale Apotheke bezogen werden. Eine Packung enthält 32 Tabletten à $150 \mathrm{mg}$ und kostet ca. $165 €$. Damit betragen die reinen Medikamentenkosten für die 12-wöchige Therapie mit Rifapentin $900 \mathrm{mg}$ plus INH $900 \mathrm{mg}$ ca. $400 €$, während die 9-monatige tägliche Therapie mit INH 300 mg ca. $105 €$ kostet. Die sichere Einnahme von bis zu 9 Tabletten als wöchentliche Einmalgabe sollte durch eine überwachte Therapie garantiert werden. Diese Kosten sind dann zu rechtfertigen, wenn die kürzere Therapiedauer zu einer besseren Akzeptanz und damit zu höheren Behandlungsraten führt.

Dr. Hilte Geerdes-Fenge, Prof. Emil C. Reisinger, Rostock 